\title{
Report of the
}

\section{LCLS Diagnostics and Commissioning Workshop* \\ University of California at Los Angeles \\ January 19-20, 2004}

\section{Organization and Program}

\author{
H.-D. Nuhn ${ }^{\mathrm{a}}$
}

\section{Program}

H.-D. Nuhn ${ }^{\mathrm{a}}$, C. Pellegrini ${ }^{\mathrm{b}}$

\section{Report Authors}

R. Bionta ${ }^{\mathrm{c}}$, W.M. Fawley ${ }^{\mathrm{e}}$, P. Emma ${ }^{\mathrm{a}}$, J. Galayda ${ }^{\mathrm{a}}$, Z. Huang ${ }^{\mathrm{a}}$, P. Krejcik ${ }^{\mathrm{a}}$, A. Lumpkin ${ }^{d}$, H.-D. Nuhn ${ }^{\mathrm{a}}$, S. Milton ${ }^{\mathrm{d}}$, S. Reiche ${ }^{\mathrm{b}}$, J. Welch ${ }^{\mathrm{b}}$, B. Yang ${ }^{\mathrm{d}}$

\author{
Local Organization \\ M. Laraneta ${ }^{b}$, S. Reiche \\ *a Stanford Linear Accelerator Center \\ Stanford Synchrotron Radiation Laboratory \\ P.O. Box 4349, Bin 69 \\ Stanford CA 94309-0210 \\ ${ }^{\mathrm{b}}$ University of California at Los Angeles \\ Department of Physics and Astronomy \\ 4-108 Knudsen Hall \\ Los Angeles, CA 90095-1547 \\ ${ }^{\mathrm{c}}$ Lawrence Livermore National Laboratory \\ 7000 East Ave. \\ Livermore, CA 94550-9234 \\ d Argonne National Laboratory \\ Advanced Photon Source \\ 9000 Cass Ave. \\ Argonne, ILL 60439 \\ ${ }^{\mathrm{e}}$ Lawrence Berkeley National Laboratory \\ 1 Cyclotron Road \\ Berkeley, CA 94720
}

* Work supported in part by the Department of Energy Contract DE-AC03-76SF00515 and Office of Science 


\section{Table of Contents}

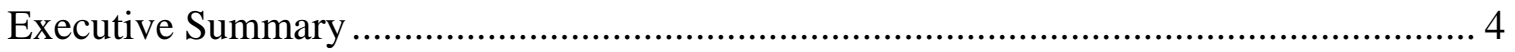

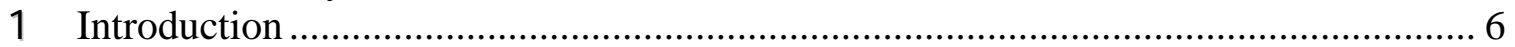

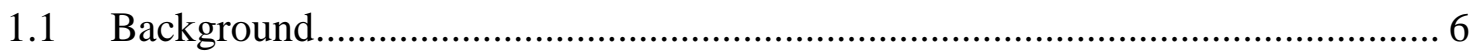

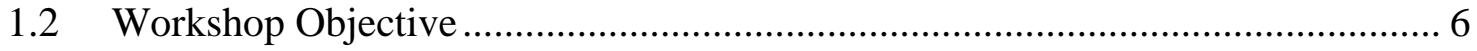

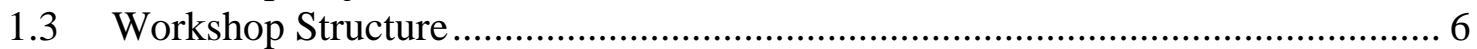

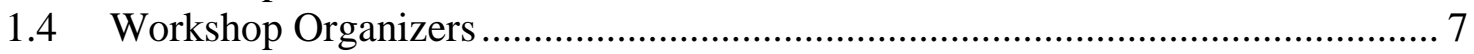

2 Charge to the Workshop........................................................................................... 7

3 LCLS Diagnostics and Commissioning ……………................................................ 7

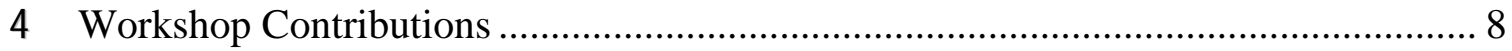

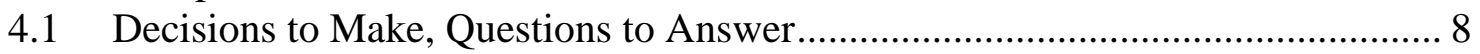

4.2 Status of Electron Beam Diagnostics .............................................................. 9

4.2.1 Electron Beam Characterization .......................................................... 9

4.2.2 Electron Beam Monitoring ………………………....................................... 9

4.2.3 Status of Individual Diagnostic Devices..................................................... 10

4.2.4 Collimation and Machine Protection System ................................................. 12

4.2.5 Feedback Implementation......................................................................... 13

4.3 X-Ray Diagnostics After the Undulator .............................................................. 13

4.3.1 Diagnostics in the FEE........................................................................... 13

4.3.2 Diagnostics in the Commissioning Diagnostics Tank .................................. 15

4.3.3 Total Energy......................................................................................... 15

4.3.4 Pulse Length............................................................................................. 16

4.3.5 Photon Spectrum ................................................................................ 16

4.3.6 Transverse Coherence.............................................................................. 16

4.3.7 Spatial Shape and Centroid Location............................................................. 16

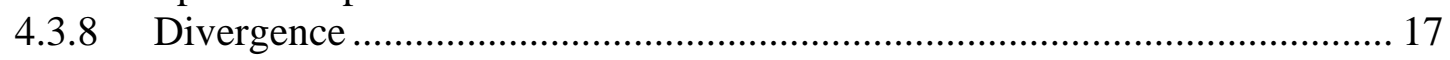

4.4 Electron Beam Control ...................................................................................... 17

4.4.1 Undulator Collimation and Protection ......................................................... 17

4.4.2 Verification of the Convergence of Beam Based Alignment (BBA) ........... 19

4.4.3 15-Å Startup for Nearly Guaranteed FEL Gain .............................................. 19

4.5 Commissioning Simulations using GENESIS 1.3......................................... 19

4.6 Future Extension of Genesis 1.3 and GINGER ................................................ 20

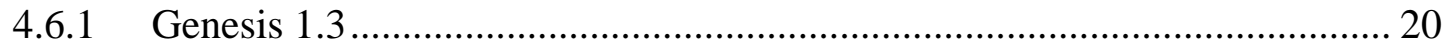

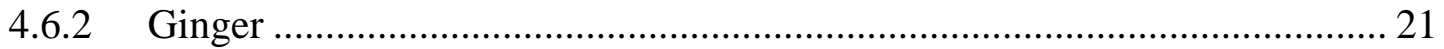

4.7 Measuring the FEL gain with Trajectory Distortion ............................................. 21

4.8 A Quick Note on the Possible Usefulness of Coherent Microbunching as a

Diagnostic in the Early Stages of the LCLS Undulator................................................ 22

4.9 A Concept For z-Dependent CXTR Microbunching Diagnostics for the LCLS 22

5 Workshop Recommendations .............................................................................. 25

5.1 No Intra-Undulator-Segment X-Ray Diagnostics in Baseline Design ................. 25

5.2 X-Ray Diagnostics Downstream of the Undulator................................................... 25

5.3 Trajectory Distortion Method to Characterize FEL Radiation vs. z..................... 25

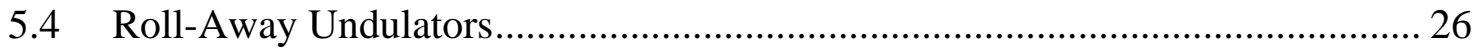

5.5 Variable Gap Undulators .................................................................................. 26 
5.6 Spontaneous Radiation as Diagnostics Tools................................................ 26

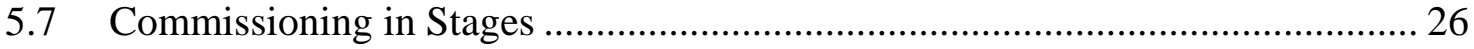

5.8 Software Commissioning Support................................................................... 27

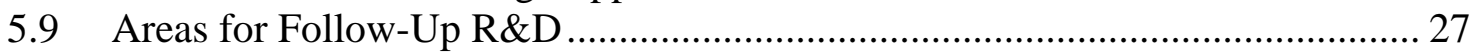

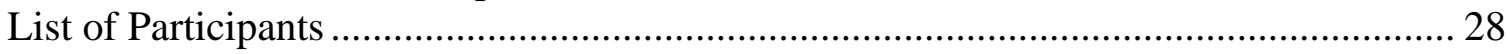

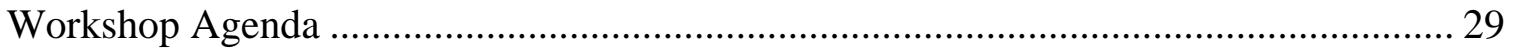




\section{Executive Summary}

The "LCLS Diagnostics and Commissioning Workshop was held at the University of California at Los Angeles (UCLA) on January 19-20, 2004 to establish the basis for a plan (published under separate cover) to commission the Linac Coherent Light Source (LCLS) undulator line with the final goal of saturating the FEL process down to the shortest fundamental x-ray wavelength of 1.5 Angstrom.

The workshop focused on requirements for the generation and characterization of $\mathrm{x}$ ray radiation during the commissioning phase including electron beam control, electron and $\mathrm{x}$-ray beam diagnostics, as well as the development of simulation codes to support commissioning.

The workshop was presented with a proposal for commissioning diagnostics and with procedures to carry out FEL commissioning towards the end of the LCLS construction phase. The workshop accepted the general plan. The charge to the workshop was summarized in the question: Will the undulator diagnostics serve commissioning and operations needs for the LCLS? There were concerns and recommendations for a number of items that are described in the following paragraphs.

The absorption rate of materials for $x$-rays in the operational range of the LCLS is very high. It has been estimated that the LCLS FEL beam is likely to melt any material with a higher $\mathrm{Z}$ than carbon at $1.5 \AA$, and all materials at $15 \AA$. This highly destructive power of the FEL radiation constitutes a problem for x-ray diagnostics along the undulator and thus for the characterization of the development of FEL radiation along the undulator, information which is essential for fully understanding the FEL process. In fact, there is presently no known solution for a survivable x-ray detector that could be used as a meaningful diagnostics in the limited space between undulator segments. An alternative method for the characterization of the development of FEL radiation along the undulator exists as described below. The workshop therefore commended to remove the intraundulator-segment $\mathrm{x}$-ray diagnostics from the baseline design, but to continue studying $\mathrm{x}$ ray diagnostics possibilities as and $\mathrm{R} \& \mathrm{D}$ activity.

There are ideas for workable solutions for x-ray diagnostics at the end of the undulator, where protection from high radiation densities can be provided through attenuators or by positioning the diagnostics components at large distances, i.e. towards the Far Experimental Hall. The main diagnostics components to be used for FEL commissioning downstream of the undulator include a CCD camera (9 microns pixel resolution, $1024 \times 1024$ pixels area) and a spectrometer.

In order to be able to measure FEL radiation characteristics, such as power and spectrum as a function of position along the undulator, but without having x-ray diagnostics available along the undulator line, a method of orbit distortion can be used, which relies solely upon diagnostics down-stream of the undulator, as discussed at the workshop. This method uses the fact that FEL gain is nearly totally suppressed if the electron beam is forced onto a large-amplitude betatron trajectory. Initial numerical simulations have shown that with a transverse displacement of one of the quadrupoles by a distance well within the range of quadrupole motion, FEL gain can be stopped and the FEL intensity at the end of the undulator is not much different from that at the position of the displaced quadrupole. The x-ray diagnostics downstream of the undulator can then be used to characterize the radiation. This method appears feasible. The increased level of 
spontaneous radiation at the end of the undulator will only slightly reduce the sensitivity to FEL radiation.

Alternatives such as roll-away undulators and variable gap undulators were discussed. The workshop found that while there would be a small benefit from having a roll-away undulator capability, this would require a considerable engineering effort. For the variable gap option there is a risk of not being able to repeatable set the $\mathrm{K}$ value to the required tolerance. The fixed gap design, for which a prototype has been constructed, has the related problem of setting the precise value of $\mathrm{K}$ when it is built. Assuming that this problem can be solved for the fixed gap undulator (and this seems to be the case), the additional cost and time required to develop a variable gap undulator with very close gap tolerances does not seem necessary at this time.

The workshop strongly recommended exploring the possibility that analysis of spontaneous radiation could characterize and improve the undulator line. If none of the methods of turning off radiation from a given $\mathrm{Z}$ location on, such as remotely-controlled roll-away undulators or variable gap undulators, can be used the options of characterizing spontaneous radiation are limited. Still it is necessary to generate detailed calculations of both the near and far field distributions of the spontaneous radiation as seen at an upstream detector.

It is important to develop software that can predict and analyze the spontaneous field distribution expected from the LCLS undulator line. Start-to-end codes are already capable of predicting the distribution of the FEL radiation. The existing codes are presently not capable of predicting the full spectrum of spontaneous radiation. The workshop therefore recommended focusing on producing data exchange interfaces between the start-to-end codes and additional radiation analysis codes that might be developed by the x-ray optics groups either from scratch or based upon existing x-ray diagnostics codes. 


\section{Introduction}

This is the report on a workshop held at the University of California at Los Angeles (UCLA) on January 19-20, 2004 to establish the baseline values for the commissioning of the LCLS FEL. This report consists of an Executive Summary, summaries of the presentations, and a list of recommendations. The workshop program is attached.

\subsection{Background}

The Stanford Linear Accelerator Center (SLAC) is leading the effort to build a FreeElectron-Laser (FEL) operating in the wavelength range 1.5-15 A. This X-ray FEL called Linac Coherent Light Source (LCLS) utilizes the last third of the SLAC Linac and is characterized by extremely high peak brightness, sub-picosecond long pulses and a fully transversely coherent radiation pulse.

The LCLS is a project of the U.S. Department of Energy (DOE) and has passed the first three critical decisions (CD-0, CD-1, CD-2a). Critical decision $2 b$ (CD-2b) the approval of the performance project baseline range is expected for June of 2004 after DOE review that is tentatively scheduled for May 2004.

\subsection{Workshop Objective}

The objective of this workshop was to establish the basis for a plan to commission the LCLS undulator line with beam with the final goal of saturating the FEL process down to the shortest x-ray wavelength of 1.5 Angstrom. The workshop covered the part of the commissioning activity that will start after the following prerequisite tasks are completed

- Undulator, Diagnostics, Shielding, Beam Dump etc. in Place

- Commissioning Without Beam for all Components Complete

The main commissioning tasks are expected to be

- Characterization of Electron Beam Up-Stream of Undulator

- Establishment of a Good Beam Trajectory Through Undulator to Beam-Dump

- Characterization of Spontaneous Radiation

- Establishment of SASE Gain

- Characterization of FEL Radiation

The commissioning plan and diagnostics are aimed at achievement of saturation of the SASE process over the LCLS operating spectrum, $1.5-15 \AA$, and verification of LCLS performance. This is distinct from the end-of-construction milestone, defined by DOE as prerequisite for approval of Critical Decision 4 (CD-4). The end-of-construction goal is expected to be one of the first milestones of the commissioning process.

\subsection{Workshop Structure}

Guidance documentation, including a draft commissioning plan, were posted to the workshop web page before the date of the workshop to aid the participants and to help keeping the workshop focused. The final version of the commissioning plan will be published independent of this report. 
The workshop opened with a presentation giving the overall charge for the workshop, followed by presentations addressing the performance of LCLS diagnostics systems. These were followed by talks that presented the results of simulations of the model cases. The workshop concluded with a discussion of the presented materials and the development of a set of recommendations.

\subsection{Workshop Organizers}

The workshop was chaired by Heinz-Dieter Nuhn (SLAC). The contact person at UCLA was Sven Reiche (UCLA). Melinda Laraneta (UCLA) was in charge of administration.

\section{Charge to the Workshop}

The Charge to the LCLS Undulator Commissioning and Diagnostics Workshop was given by J. Galayda as the following set of questions:

"Will the Undulator Diagnostics Serve Commissioning and Operations Needs for the LCLS?"

- Commissioning

o Can diagnostics be used to troubleshoot the new hardware?

o Can diagnostics be used to guide path to saturation?

- Operations

o Will the diagnostics permit simple and speedy troubleshooting?

o Reliability/Availability goals of the LCLS will be those of a light source

- X-ray diagnostics are crucial

o Can the diagnostics survive at high power?

o If not, are we placing too heavy a reliance on data taken with low charge?

- What are the alternatives?

o Variable gap?

o Rollaway undulators?

- Do we have redundant diagnostics capability where appropriate?

o Diagnostics that check the diagnostics

\section{LCLS Diagnostics and Commissioning}

The LCLS commissioning has been discussed over the last several years. Many unsolved questions remain. Some of the identified main challenges are: radiation protection of magnetic material during commissioning and operation, and the development of detectors that can withstand the heat load. What is needed is a compilation of descriptions of detector types available for commissioning (both existing and under development), including electron detectors upstream of the undulators, electron and $\mathrm{x}$-ray detectors along the undulator electron and x-ray detectors after the undulator, decision on how to measure the FEL gain curve.

Past experience has show that it is important to support the commissioning effort 0with computer simulations: We believe that it is desirable to enhance the existing codes to include the simulation of $\mathrm{x}$-ray diagnostics. 


\section{Workshop Contributions}

This section summarizes individual workshop contributions that were presented both as invited (other than the charge, introduction and summary talks) and contributed talks during the workshop itself as well as notes submitted after the workshop. Sections 4.1 to 4.8 are summaries of presentations (J. Welch (4.1), P. Krejcik (4.2), S. Milton/B. Yang (4.3), R. Bionta (4.4), P. Emma (4.5), S. Reiche (4.6), W. Fawley (4.7), and Z. Huang (4.8)) given at the workshop. Section 4.9 (W. Fawley) and 4.10 (A. Lumpkin) are later contributions based on discussions during the workshop.

\subsection{Decisions to Make, Questions to Answer}

For the design of the undulator system to proceed, (including diagnostics, undulator magnets, vacuum chamber, movers, supports, etc), some key decisions have to be made as to what capabilities to include. We must include a minimum set of capabilities so that the undulator can reasonably be expected to work. We may also want to include additional capabilities that give us performance flexibility or improve our ability to diagnose and measure various beam properties. Some of the key design decisions are listed below:

- With regards to comb, canted poles, shimming: Which method will we use to obtain the proper K value?

- Do we put x-ray diagnostics in the undulator line? Will they survive?

- Should the segments be designed to be remotely removable?

- Do we need longitudinal phase adjustment?

Including all capabilities listed above is probably not the best answer. Such a choice would generate substantial cost and schedule increments, severely limit design flexibility and lead to compromises that may actually decrease the performance of the overall system. Below is a list of questions whose answers would help us make the best choice of system capability.

- How would the comb $\mathrm{K}$ adjustment mechanism be used in commissioning and tune up?

- How would phase adjustments control at the end of each segment be used in commissioning and tune up?

- If an undulator segment is removed what is the resulting phase error and how do we correct for it?

- Can we use segment removal as a diagnostic? What will it actually diagnose?

- What kind of pulse-to-pulse variability should we expect in x-ray intensities, both from SR and FEL radiation, before and after saturation?

- What is the minimum detectable SR and FEL radiation as a function of distance along the undulator, and downstream of the undulator, taking into account losses of SR to the vacuum chamber, at both high and low electron beam energies?

- For each segment, what is the sensitivity of the total FEL output power to phase error from that segment, including large phase errors, at low and high energies, for design and "relaxed" commissioning parameters? 
- For locally imposed trajectory bumps, chosen at different positions along the undulator what is the sensitivity of the output FEL radiation to the bump amplitude, assuming an otherwise perfect undulator with normal cosh/cos transverse field dependence, at both low and high energies?

- What is the pulse-to-pulse pointing stability expected for the FEL radiation?

- What is the effect upon the FEL spot if the undulator is perfectly curved with a constant radius of $7500 \mathrm{~km}$ for both low and high energies? Such a curve will be within the nominal tolerance of 2 microns over 10 meters.

\subsection{Status of Electron Beam Diagnostics}

The electron beam diagnostics for LCLS are reviewed here in the context of undulator commissioning plans. The two areas of focus are the electron beam commissioning in readiness for undulator tests and the anticipated use of electron beam diagnostics during FEL tuning

\subsubsection{Electron Beam Characterization}

Measurement of 6D phase space takes place before the beam enters the undulator to confirm that it meets the requirements for SASE operation. The transverse emittances are measured in the $\mathrm{x}$ and $\mathrm{y}$ planes only. The round beams from the injector and the absence of any appreciable source of coupling do not call for any special diagnostics to measure the skew component of the beam. The bunch length and energy spread measurements complete the 6 dimensions of phase space.

The beam centroid measurements comprise of precise, relative measurements of beam energy and beam position. The absolute energy of the electron beam is most precisely given by the fundamental photon wavelength in the undulator. The electron energy is also measured in a magnetic spectrometer in both the LTU before injection into the undulator and in the dumpline at the exit of the undulator.

Beam energy and position measurements emphasize sensitivity to relative changes rather than absolute measurement. Beam based alignment will be used for straightening the electron beam in the undulator.

\subsubsection{Electron Beam Monitoring}

Changes in the beam properties provide information on the beam dynamics in the undulator. Changes in beam energy due to Incoherent Synchrotron Radiation (ISR) and changes in the energy spread due to SASE will be resolvable at the dumpline energy spectrometer. The microbunching that accompanies the SASE process is still being investigated as to whether it is observable.

Electron beam monitoring will also observe drifts in the upstream electron beam parameters and feedback systems will be employed to counteract these drifts. The feedbacks will operate at the full beam rate of $120 \mathrm{~Hz}$ and should significantly suppress beam motion up to $10 \mathrm{~Hz}$ in bandwidth. 


\subsubsection{Status of Individual Diagnostic Devices}

\section{Beam Position Monitors}

In the injector, linac, and LTU, stripline monitors will be used. The required specified resolution is 5 microns for bunch charges in the range 0.2 to $1 \mathrm{nC}$. A new BPM processor module will be developed to work with existing linac hardware to achieve this specification. A feasibility study has been made indicating that the resolution is achievable, but no design work has been done on these modules.

The new BPMs required for the injector and LTU will be built in a similar style to the existing linac and FFTB stripline BPMs. The only significantly different linac BPMs are those for each of the bunch compressor chicanes that need a larger aperture to accommodate the spread-out beam in those locations. Their requirements are 20 micron resolution with a $3 \mathrm{~cm} \times 10 \mathrm{~cm}$ aperture.

The last 8 BPMs in the LTU are to be made redundant with 8 cavity style BPMs.

\section{Cavity Beam Position Monitors in the Undulator and LTU}

R\&D at SLAC by Steve Smith et al. has concentrated on both C-band and X-band cavity structures. A collaboration with the ATF (KEK, Japan) has demonstrated resolution better than 0.2 microns. Specific designs for LCLS applications have not yet started.

\section{BPM Controls Issues}

LCLS operation requires timing pulse identification. This will allows all BPMs to be read on the same beam pulse, which is critical for a single pass FEL machine since each pulse is potentially different.

By the same token $120 \mathrm{~Hz}$ readback of the BPMS is also required.

In order to diagnose the cause of interruptions to the beam operation it is necessary to ring buffer all BPM readings, extending back last 1000 pulses. This will allow MPS trips, for example, to be traced to an orbit excursion. Stability can be monitored by archiving RMS orbit jitter every 1000 pulses.

The BPM orbit acquisition software must be linked to the optics model to allow for real-time orbit fitting displays.

\section{Beam Size Monitors}

Wire scanners will use the basic SLC design which is essentially noninvasive to beam operation.

The technical challenges are

- Small beam size dictates small wire

- Range of beam charge $0.2-1 \mathrm{nC}$,

- compromise between signal to noise and saturation

- Signal to noise from linac dark current

- Low beam charge operation dictates high $\mathrm{Z}$ wire material

- Beam loss considerations in front of undulator dictates low Z 
Groups of 4 wire scanners are used for emittance reconstruction. They measure the average, projected emittances in $\mathrm{x}$ and $\mathrm{y}$. For undulator commissioning, the matching section at the end of the LTU verifies emittance and beta match at undulator entrance.

However, no room in the dump beam line for a zero dispersion location, so it will not be possible to fully monitor the emittance at the exit of the undulator.

\section{Profile Monitors}

Except at the injector, we will use thin foil OTR screens to image the beam on a single shot. Although we could potentially record every shot individually at $120 \mathrm{~Hz}$ we will limit the scope of the data acquisition scheme to approximately $10 \mathrm{~Hz}$ maximum capture rate.

Technical challenges for the profile monitors are:

- Small beam size requires precision, remote optics + digital video (\$’s)

- Stretching a thin, low Z foil flat and avoiding punctures

The OTR screen does measure single pulse $\mathrm{x}$ and $\mathrm{y}$ beam profiles. It acts as emittance spoiler, but the scattering from the foil is small enough that the beam can still be transported to the dump.

Profile monitors will measure the energy spread profile in high dispersion locations:

- Injector inflector, chicanes, LTU dogleg, dump line

The advantage of OTR screens is that we can block synchrotron radiation from adjacent bends with polarizers.

\section{Slice Parameters from Transverse RF Deflecting Cavity}

Single pulse slice emittance diagnostics are only possible with a profile monitor, but these are invasive since they are done in conjunction with the transverse RF deflecting cavity. The OTR screen down stream of the TCAV can be used in conjunction with a quadrupole scan to measure slice emittance in one plane only (horizontal). A second transverse cavity would be required if slice emittances in both planes are to be measured.

The slice energy spread will be measured at an OTR screen downstream of the TCAV where the horizontal dispersion is large, such as in the DL2 dogleg of the LTU.

\section{Profile Monitors for Synchrotron Radiation}

A single shot, noninvasive projected energy spread measurement can be made from the synchrotron radiation generated from a vertical chicane wiggler in a horizontal dispersion region in the DL2. The ISR strikes an off-axis screen and the optical resolution is set by the divergence of the x-rays. The resolution is maximized by filtering out low energy x-rays with a foil and using a thin fluorescent crystal. However, this is not likely to be able to resolve the extremely low incoherent energy spread in the LCLS beam, but it would serve as a simple way of detecting gross errors in the machine settings.

\section{Beam Phase Monitors}

These employ linac style S-band monitor cavities and can measure pulse-to-pulse phase jitter. Since they are also subject to thermal drift they can not be used for feedback control of the beam phase. However, thermal stabilization technology (as required for the undulator) may make this possible in the future. The beam phase can be measured w.r.t. the RF distribution or the laser from the injector or at the experiment. 


\section{Bunch Length Monitors}

Several devices are planned for bunch length measurement and monitoring. Each of the devices has a unique feature that complements the others during machine operation. The devices differ in terms of invasiveness, absolute versus relative measurement, average versus single pulse measurement, and sensitivity to microbunching instability signatures.

Measurement of subpicosecond bunch profiles is a new technology and each of the devices reported here are at an $\mathrm{R} \& \mathrm{D}$ stage and require significantly more development. A comparative summary table of the planned bunch length monitors is given below.

Table 1: Comparison of the capabilities of the different bunch length monitoring devices.

\begin{tabular}{|l|l|l|l|l|l|}
\hline Device & $\begin{array}{l}\text { Invasive } \\
\text { measurement }\end{array}$ & $\begin{array}{l}\text { Single shot } \\
\text { measurement }\end{array}$ & $\begin{array}{l}\text { Absolute or } \\
\text { relative } \\
\text { measurement }\end{array}$ & $\begin{array}{l}\text { Timing } \\
\text { measurement }\end{array}$ & $\begin{array}{l}\text { Detect } \\
\text { micro- } \\
\text { bunching }\end{array}$ \\
\hline $\begin{array}{l}\text { RF Transverse } \\
\text { Deflecting Cavity }\end{array}$ & $\begin{array}{l}\text { Yes: Steal 3 } \\
\text { pulses }\end{array}$ & No: 3 pulses & Absolute & No & No \\
\hline $\begin{array}{l}\text { Coherent Radiation } \\
\text { Spectral power }\end{array}$ & $\begin{array}{l}\text { No for CSR } \\
\text { Yes for CTR }\end{array}$ & Yes & Relative & No & Yes \\
\hline $\begin{array}{l}\text { Coherent Radiation } \\
\text { Autocorrelation }\end{array}$ & $\begin{array}{l}\text { No for CSR } \\
\text { Yes for CTR }\end{array}$ & No & Relative & No & No \\
\hline $\begin{array}{l}\text { Electro Optic } \\
\text { Sampling }\end{array}$ & No & Yes & Absolute & Yes & No \\
\hline
\end{tabular}

\subsubsection{Collimation and Machine Protection System}

\section{Collimation}

Movable energy collimators are used in each chicane as a diagnostic to distinguish the head and tail of the bunch and will be adaptable for foil slits to be used for femtoslicing.

The final energy collimation is done with a pair of adjustable collimators in the dogleg bend DL2 of the LTU. Transverse collimation is done with three $\mathrm{x}$ and $\mathrm{y}$ adjustable collimators in the matching section of the LTU at two betatron phases and one clean-up in each plane

\section{Beam Loss Monitors}

Protection Ion Chambers will be installed at each collimator location to detect errant energy and orbit conditions. A distributed loss monitor, such as a PLIC cable or a fiber optic system, will be placed in the undulator gap. Complementary beam loss monitors will also be installed in the inter undulator gaps.

\section{Beam Rate Limiting}

A single bunch beam dumper (SBBD) will be installed in the LTU to select the rate at which beam pulses are sent to the undulator. The linac beam up to the dog-leg bend in the LTU can be maintained at $120 \mathrm{~Hz}$ which is favorable for upstream stability and feedback operation. The SBBD will be triggered by beam loss conditions through the MPS system. 
A failure in the pulsed magnet will turn off beam at the gun. A tune-up dump at end of the LTU will receive a maximum of $10 \mathrm{~Hz}$.

In addition to trips from beam loss, the SBBD will also be triggered by invalid readings from the undulator: vacuum, magnet movers, BPMs, launch orbit feedback failing, magnet power supplies for some key elements.

\subsubsection{Feedback Implementation}

Numerous feedback loops will be implemented through out LCLS for pulse-to-pulse control of orbit position, angle and energy. The operation of these loops has been demonstrated at SLC. At LCLS the design of these loops will require integration into the new control system architecture. A more challenging loop is the feedback control of bunch length after each of the bunch compressors. These loops will use the relative signal strength from CSR $\mathrm{THz}$ spectral power measurement. Although this has been demonstrated at SPPS with dither feedback to minimize bunch length the LCLS bunch compressors will need power measurement at several $\mathrm{THz}$ wavelengths to tune to arbitrary bunch lengths. This will need further R\&D effort.

\subsection{X-Ray Diagnostics After the Undulator}

\subsubsection{Diagnostics in the FEE}

The LCLS Front-End-Enclosure (FEE) is a 40-meter-section of tunnel between the end of the undulator and the near hall where the electron beam is separated and dumped and where initial diagnostic and beam conditioning equipment will be located. It is expected that the diagnostics in the FEE will be operational shortly after the completion of the Undulator installation and therefore will be available for the first light. The FEE beam line has the following components: 1) a fast close valve, 2) a pair of horizontal and vertical slits, 3) a Diagnostics Tank containing imaging diagnostics, 4) a gas attenuator system 5) a solid attenuator, 6) another Diagnostics Tank, and 7) a final set of horizontal and vertical slits. The two sets of slits are to allow the $\mathrm{x}$-ray beam to be delivered to the experimental halls, stripped of much of the spontaneous radiation component. The solid attenuator is a block of low $\mathrm{Z}$ material that can be inserted into the beam to attenuate the FEL radiation. The gas attenuator is used at lower photon energies that would damage the solid attenuator. The function of the diagnostics before and after the attenuator is, in the long term, to monitor the attenuator and slits, and, in the short term, to measure the beam intensity and footprint in the very early commissioning stages of the LCLS.

To mitigate some of the technical risk, and to span the full range of FEL photon energies, the imaging diagnostics in the Diagnostics Tanks come in three varieties with partially overlapping operating regimes. Each diagnostic tank contains the following systems:

- Direct Scintillation Imager An insertable, high-resolution scintillator viewed through a microscope objective by a CCD camera for measuring spatial distributions and for alignment and focusing of optical elements. The scintillator is a 100 micron thick Ce doped YAG or LSO crystal. The camera can be configured to have a FOV of 2 to $10 \mathrm{~mm}$ with a spatial resolution of 2 to 20 microns 
Placed directly in the beam, the direct scintillation imager will saturate even in response to one pulse from the spontaneous at modest power levels. A set of low$\mathrm{Z}$ foils in front of the camera will attenuate the beam enough to allow unsaturated imaging of the spontaneous radiation pattern at full power.

Direct exposure to the FEL beam will damage the crystal in one pulse. At FEL photon energies above $4000 \mathrm{eV}$ thicker blocks of $\mathrm{B}_{4} \mathrm{C}$ and $\mathrm{Be}$ can be used to attenuate the FEL beam by a factor of $10^{-4}$, enough to image it with the Direct Scintillation Imager. In this case the high spatial resolution of the camera will allow the FEL to be spatially separated from the spontaneous. At lower photon energies the solid attenuators will suffer damage and the gas attenuator must be used to lower the intensity of the beam.

The chief technical risk is in our understanding of the photon attenuation and transport through the solid attenuators and their damage thresholds at the FEL fluence levels. Although relatively simple, the Direct Imager will totally block the beam, limiting its usefulness.

- Indirect Imager The Indirect Imager utilizes a thin foil of a low Z Be to act as a beam splitter to partially reflect a portion of the beam onto the crystal of a (Direct) imaging camera which remains out of the beam. The reflected intensity can be adjusted by changing the angle of incidence. A reflectivity of $10^{-4}$ can be obtained with an incident angle of $1^{\circ}$ at $8 \mathrm{keV}$ and an incident angle of $>2^{\circ}$ at $0.8 \mathrm{keV}$.

At the higher energies the Beryllium foil will be transparent and a significant fraction of the beam will be transmitted downstream. This will allow pairs of these detectors to monitor the radiation going into and out of another instrument.

If indeed the reflector has a higher damage threshold than a normal incidence optic, the indirect imager will work at all FEL photon energies although it will only be transparent at the highest energies. Another foreseeable problem with this concept is the background $\mathrm{x}$-ray radiation impinging on the crystal due to Compton scattering of the FEL beam by the Be foil, and any fluorescence from an oxide layer on the foil surface.

- Micro-Strip Ion Chamber The third detector measures the ionization produced by the FEL as it traverses a small amount of gas. The gas is trapped between two differentially pumped sections so there can be no windows or other solids in the beam. By segmenting the anodes and cathodes into strips parallel to the beam direction, we hope to obtain limited spatial information on the profile of the beam sufficient to separate the FEL from the spontaneous.

The microstrip ion chamber will provide spatially resolved profile data at all FEL photon energies. The utility of this device depends on the performance of the 
microstrip anode and cathodes arrays. Quantitative interpretation of the data also depends on our understanding of the physics of the gas interactions at FEL fluence levels.

\subsubsection{Diagnostics in the Commissioning Diagnostics Tank}

The "Commissioning Diagnostics" tank will be located in the third hutch of the Near Experimental Hall (NEH), approximately $65 \mathrm{~m}$ from the end of the Undulator. Because of its location at the end of the NEH these diagnostics will probably not be in place for several months after the first light, and in any case will require a slightly more stable and controlled FEL operation. Most measurements will have to be done with an attenuated FEL beam to prevent damage to the instrumentation.

The "commission" diagnostics are intended to measure the basic FEL performance parameters during commission and are allowed to be "intrusive". The goals of the commissioning diagnostics are to measure

1. Total pulse energy

2. Pulse length

3. Photon energy spectrum

4. Transverse coherence

5. Spatial shape and centroid location

6. Divergence

The commissioning diagnostic tank has a central optical rail and stages for apertures, optics, a Direct Scintillation Imager, and other hardware common to these measurements. The specific equipment needed to perform specific measurement such as the calorimeter, will be set up, and taken down as needed.

\subsubsection{Total Energy}

It is desirable to measure the FEL pulse energy utilizing calorimetric techniques to avoid any reliance on the theory of photon-atom interactions at LCLS intensities.

The calorimeter has a small volume x-ray absorber which absorbs all of the x-ray energy resulting in a rapid temperature rise. The heat capacity and mass of the absorber determine the temperature rise. For a 1\% measurement, the thickness of the absorber must be at least 5 mean free path lengths in order to capture better than $99 \%$ of the x-ray energy. The sensor measures the temperature rise of the absorber. The thermal mass of the sensor be small compared to the absorber. The heat in the absorber is conducted through the thermal semiconductor to the heat sink. The purpose of the thermal semiconductor is to delay the heat transfer from the absorber to the heat sink long enough to measure the temperature rise in the absorber. The heat sink is held at a constant temperature. The energy deposited by the $\mathrm{x}$-ray is conducted into the heat sink before the next x-ray pulse. The thermal conductivity of the absorber, the thermal conductivity of the thermal semiconductor, the geometry of the absorber, and the geometry of the thermal semiconductor control the rate at which the heat in the absorber is conducted to the heat sink.

For $8 \mathrm{keV}$ operation the absorber will be a Si cylinder $0.5 \mathrm{~mm}$ in diameter and $0.5 \mathrm{~mm}$ thick. The $0.5 \mathrm{~mm}$ thickness is $>5$ attenuation lengths and the $0.5 \mathrm{~mm}$ diameter nicely accommodates the $\sim 340$ microns FWHM diameter of the $8 \mathrm{keV}$ FEL at the 
position of the commissioning diagnostics tank. The dose at $8 \mathrm{keV}$ to $\mathrm{Si}$ in this position is $0.12 \mathrm{eV} /$ atom, which is acceptable for a simple absorber.

For $0.8 \mathrm{keV}$ operation the absorber will be a Be disk $3 \mathrm{~mm}$ in diameter and $>25$ microns thick since the dose to $\mathrm{Si}$ at this wavelength is too high. The $3 \mathrm{~mm}$ diameter is necessary to contain the $0.88 \mathrm{keV}$ beam whose diameter at this position is $1.9 \mathrm{~mm}$ FWHM.

The calorimeter will be positioned on the optics stage in the commissioning tank allowing it to be aligned utilizing the rear imaging detector

\subsubsection{Pulse Length}

Measuring the $233 \mathrm{fs}$ pulse length is perhaps the most challenging measurement at the LCLS. Several concepts have been proposed, all involving a medium which modulates an external laser beam when exposed to the x-ray FEL. The method we have chosen to baseline is illustrated. The beam from a $1500 \mathrm{~nm}$ CW laser is split and made to pass through the two arms of an interferometer patterned in GaAs on a substrate. X-rays impinging on one of the arms changes its index of refraction causing a modulation in the $\mathrm{CW}$ beam after it is recombined. The modulation of the CW beam is in principle of the same duration as the X-ray pulse and can be measured with a streak camera to $>100 \mathrm{fs}$. To achieve better temporal resolution, the modulated CW beam is sent through a time microscope which stretches the pulse by a factor of 2 to 100. The stretched pulse length is then measured with the streak camera.

The device can also be used to synchronize an external laser pulse with the x-ray beam. This is accomplished by feeding the external pulse through the time microscope alongside of the x-ray modulated $\mathrm{CW}$ pulse and measuring both on the same streak camera.

\subsubsection{Photon Spectrum}

The commissioning diagnostic tank is converted into a spectrometer by adding a crystal at $8 \mathrm{keV}$ or a grating at $0.8 \mathrm{keV}$. In either case the optic is curved so as to focus onto the $\mathrm{x}$-ray sensitive region of a fast readout linear array.

\subsubsection{Transverse Coherence}

We will measure the transverse coherence in the commissioning diagnostics tank using the setup shown in the figure that employs an array of double slits with constant slit width but different slit spacings. The slits sample the beam in two places and the resulting diffracted beams interfere with each other at the position of the detector.

At $0.8 \mathrm{keV}$ the slits will be assembled from polished sticks of low-Z material such as $\mathrm{B}_{4} \mathrm{C}$ or $\mathrm{Si}$ held apart by spacers. The higher resolution slits for $8 \mathrm{keV}$ will be manufactured by the sputter-sliced method or from an array of fibers.

\subsubsection{Spatial Shape and Centroid Location}

The spatial shape and centroid location of the FEL beam will be measured on a pulseby-pulse basis by the Scattering Foil Detectors located in the facility diagnostics tanks distributed along the beam lines. 


\subsubsection{Divergence}

This measurement is performed at $8 \mathrm{keV}$ using the Scattering Foil Detectors located along the beam line. The measurement is performed at $0.8 \mathrm{keV}$ using the LCLS Segmented Ion Chambers located along the beam line.

\subsection{Electron Beam Control}

\subsubsection{Undulator Collimation and Protection}

Devices for protecting the undulator magnets from exposure to radiation generated by the electron beam will be located between Dogleg-2 (DL2) and the last undulator module.

\section{Energy Collimation}

Devices at DL2 measure linac energy and prevent off-energy electrons from reaching the undulator. Two beam collimators will be used to limit particle energy halos and to protect against all accidental changes in linac energy including klystron drop-outs. These collimators will be located at each of the two high dispersion points at the beginning and at the end of DL2. They will have a bore diameter of $10 \mathrm{~mm}$ and will limit the energy range to $+/-4 \%$. Simulations with ELEGANT show that the proposed setup will limit the maximum excursion of particles in the undulator vacuum chamber to $+/-0.5 \mathrm{~mm}$, if the rest of the beamline is unperturbed.

\section{Transverse Phase Space Collimation in the Diagnostics Section}

Further downstream of the dogleg is the diagnostics section, which is designed to have the same beta-functions for all operational energy values. The diagnostics area is made up of 3 regular FODO cells with a phase advance of 45 degrees per cell. There will be 4 movable collimators with $4 \mathrm{~mm}$ nominal bore diameter in this section, 2 each for horizontal and vertical phase space limitation. The collimators in each pair will be spaced by 90 degrees of betatron phase advance relative to each other. They will be located close to the maxima of the beta functions for maximum efficiency. The collimators will limit maximum betatron amplitudes inside the ideal undulator to $+/-2.5 \mathrm{~mm}$.

The transport of a beam halo at a large emittance of 4000 microns through the twophase $\mathrm{x}$ - and $\mathrm{y}$-collimation system in the diagnostics area has been simulated. The collimator bore diameter used in the simulation is $4 \mathrm{~mm}$. The maximum excursion inside the undulator for those electrons that pass through the collimator system is about $2 \mathrm{~mm}$. These results apply to all operational energies. The simulations did not include scattering of electrons at the collimator edge, which could reach larger excursions.

There is an option to add a third phase of collimators right after the diagnostics section, i.e., at the beginning of the matching section. The phase advance between those third phase quadrupoles and the corresponding second phase quadrupoles are still fairly close to 90 degrees. These third phase collimators will clean up potential edge scattering on the primary collimators, but may not be required. 
The collimation system requires electromagnetic quadrupoles in between collimators in order to collimate in all transverse phase space dimensions. The undulator protection by the collimator system will not be sufficient if one or more of these quadrupoles fail. An interlock system will be in place to abort the beam in the event that the quadrupole magnets get out of tolerance. The response time of the quadrupoles is slow. A failure can be detected early enough to stop beam operation and prevent damage to the undulator.

In addition to the collimators, there will be at least 4 wire scanners and 4 RF Cavity BPMs in the diagnostics section. The matching section, which follows the diagnostics section, will have 2 additional cavity BPMs.

\section{Diagnostics and Protection Around the Undulator}

There will be a removable tune-up dump upstream of the first undulator and a beam abort system after dogleg DL-2.

At the beginning and at the end of the undulator line there each will be a beam current monitor, which will be used as backup diagnostics to detect major beam loss inside the undulator at the $5 \%$ level.

In front of every undulator segment there will be a beam loss monitor that will be interlocked to abort the beam starting at the next $120 \mathrm{~Hz}$ pulse.

Beam loss detectors also include a pair of long distributed beam loss monitors that will run inside the undulator gap on each side of the vacuum chamber.

\section{Undulator Quadrupole Offset Errors}

Beam losses generated by quadrupole-offset errors have been simulated with the ELEGANT code, as well. The result is that chamber hits are unlikely. These results also apply to all operational energies.

Kicks from quadrupole offsets get larger for lower energy. In the LCLS undulator the beta function gets smaller with lower energy. The maximum amplitude of the betatron oscillation, $\mathbf{x}_{\max }$, is proportional to the beta function and to the kick angle. Therefore, the trajectory produced by most quadrupole-offset patterns should have a very weak dependence on energy.

A maximum offset error in a single quadrupole will generate a betatron oscillation with a beam-centroid amplitude of $1.2 \mathrm{~mm}$. For offset errors of two consecutive quadrupoles at opposite sign, i.e., same kick direction, the maximum excursion is about $2.4 \mathrm{~mm}$.

Due to the alternate sign of the quadrupole strength from one quadrupole to the next, error conditions, where any number of consecutive quadrupoles has a maximum offset error in the same direction, will produce very little excursion because of cancellation.

Random quadrupole offset errors have a low probability of generating excursions above $2.5 \mathrm{~mm}$. From 100 randomly selected quadrupole offset distributions only 2 exceed $+/-2.5$ mm excursions.

There is a number of certain systematic offset errors combinations that will generate excursions larger than the vacuum chamber radius. These combinations include the situation where more than two quadrupole offset errors in a row at maximum kick angle are going in the same direction, i.e., alternate direction of quadrupole displacements. 


\section{Setup of Initial Launch}

During the setup of the initial launch condition it is possible to create trajectories in the undulator with excursions larger than the vacuum chamber radius if the BPM readings upstream of the undulator are corrected too tightly. This problem can be avoided by limiting the corrector strength, i.e., by not trying to follow corrector misalignments too closely.

\subsubsection{Verification of the Convergence of Beam Based Alignment (BBA)}

After the BBA procedure has been initially applied 2-3 times, it will be very useful to verify its convergence. This verification can be done during the procedure itself. As the procedure is iterated, the trajectory sensitivity to large energy changes should be reduced with each application. Initially, depending on the initial quadrupole misalignment levels (50 $\mu \mathrm{m}$ rms assumed here), the trajectory will change on the order of $500 \mu \mathrm{m}$ as the energy changes from $14 \mathrm{GeV}$ down to $4.5 \mathrm{GeV}$. After 3 passes of BBA, this sensitivity should be reduced to $<50 \mu \mathrm{m}$. Note that since the BPM offsets will also be known to a high degree of precision (2-3 $\mu \mathrm{m} \mathrm{rms}$ ), the $4.5-\mathrm{GeV}$ trajectory can be straightened simply be carefully steering the BPM readings back to zero, allowing FEL commissioning at long wavelength.

The earth magnetic field, if not corrected, has a noticeable effect on the result of the BBA procedure. A transverse field component of 0.2 Gauss, constant over the undulator length, causes a significant degradation of the BBA results which may not be tolerable. A field of 0.1 Gauss is, however, acceptable. This limitation might be corrected by shimming the undulators in their proper orientation with the same field present, but in any case must be taken seriously as a potential problem.

\subsubsection{5-Å Startup for Nearly Guaranteed FEL Gain}

The requirement to beam quality at the $15-\AA$ and of the wavelength range are low compared to those for 1.5- $\AA$. Saturation can be achieved with an emittance of 4 microns at $0.5 \mathrm{nC}$ and a peak current of $1 \mathrm{kA}$. Requirements for the trajectory straightness are reduced such that a relative resolution of the undulator BPMs of 6 microns is sufficient.

\subsection{Commissioning Simulations using GENESIS 1.3}

In order to model the SASE FEL experiment in numerical simulations it is essential to specify the beam properties, i.e., mean energy, energy spread, beam size, Twiss parameters, etc., for at least 20 positions along the bunch with an optimum of 150 positions, which correspond to one input parameter set for each cooperation length. Beam emittance and current have the strongest impact on the FEL performance and can render the output of the simulation meaningless if their values do not lie within the tolerances of $100 \mathrm{~A}$ or 0.2 microns for current and emittance, respectively. (These tolerances were derived from a change in the output power of one order of magnitude at the end of the undulator.)

Full time-dependent SASE simulations for the LCLS are CPU-time-expensive (several days of calculation on a single processor computer). However, the FEL performance can be estimated in about $1 \%$ of the SASE run time, using the FEL amplifier model. About 200 slices are independently seeded with an external radiation field as the 
equivalent to the start-up signal in the SASE process given by the initial fluctuation in bunching factor. At the current time, GENESIS 1.3 lacks the capability to individually seed each slice with different power levels, but an implementation of M. Xie's formula to estimate that power level for a given input parameter set for each slice is straightforward to implement.

FEL codes are highly optimized for frequencies within a small bandwidth around the resonant frequency. In addition, the spatial discretization of the radiation field cuts off high modes with strong diffraction. These FEL codes are incapable of calculating the full spectrum and angular distribution of the spontaneous radiation. However, this information is essential to determining the noise level for the X-ray diagnostic. Analytical estimates are only available for the far field zone of point like radiation sources, a model which does not well represent the LCLS. Currently, a code is under development at UCLA to overcome this limitation.

Even an estimate for the spontaneous radiation shows that a distance of several gain lengths from the beginning of the undulator is required to obtain an FEL signal larger than the spontaneous background signal. The total distance depends on the applied cut in frequency and space, but even under best circumstances the length is longer than $10 \mathrm{~m}$. Thus, no field information from the first three undulator modules can be obtained to optimize the tuning/position of these modules. Simulations have also shown that a localized error in the undulator position and field is only detectable right after the module. In addition, the optimization of the undulator field strength has local maxima in the FEL output power, which does not correspond to the overall best performance.

\subsection{Future Extension of Genesis 1.3 and GINGER}

The two main computer codes used for the LCLS project in start-to-end simulations to simulate the full FEL process including simulations are Genesis 1.3 (author S. Reiche, UCLA) and GINGER (author W.M. Fawley, LBNL).

\subsubsection{Genesis 1.3}

- A quick estimate of the FEL performance can be obtained, when the FEL is modeled by independent FEL amplifiers for each beam slice. Spontaneous radiation is excluded and only the seeding field is amplified. The power level depends on the electron slice parameters and can be estimated with analytical formulae. Currently, only constant seeding power is supported, which yields wrong results when the beam parameters vary along the bunch. Genesis 1.3 will be extended to automatically set the seed power to the shot noise power level, using Ming Xie's analytical formula.

- The execution speed will be improved by porting the code to a parallel architecture, using the MPI library.

- The code will be extended to support higher harmonics. The latest version of Genesis already provides the correct statistics in the bunching factor for the higher harmonics.

- Genesis 1.3 currently models drift spaces as a virtual undulator to provide a solution for phase matching between two modules. However the drift lengths are restricted to multiples of the integration step size, which is typically equal to the undulator period. The extensions to Genesis 1.3 will provide an alternative solver, 
where the particle solver is optimized for drifts and quadrupoles, but supporting arbitrary integration step sizes. The radiation field is advanced to the beginning of the next module by solving Huygen's integral.

- Smaller programs will streamline the interface between other programs (e.g. converting the output of Elegant into Genesis-1.3-conform input decks) or actual measurements.

\subsubsection{GINGER}

- GINGER is currently parallelized and runs efficiently in multiprocessor environments, including the massively-parallel IBM-SP at DOE's National Energy Research Supercomputer Center (NERSC)

- GINGER currently can fully utilize ELEGANT macroparticle phase space information (generally in SDDS format) for full time-dependent runs. Furthermore, in FY03, a "segment"-mode capability was added which allows the simulation of a temporally "long" beam pulse to be broken into a number of smaller runs.

- Due to the needs of LBNL and other labs, such as MIT/BATES, that are utilizing GINGER, it is hoped that the coding for following harmonic emission will be inserted in the code during FY04.

- GINGER currently has 3D modeling of electron macroparticle motion but only 2D (i.e. $r$-z) resolution of the radiation field. In a longer (human) time frame, there are plans to extend the radiation modeling to 3D by use of an azimuthal multipole decomposition as was done in the past with the FRED-3D code. In general, it should be only necessary to include harmonics through $\mathrm{m}=3$ or $\mathrm{m}=4$.

- In the next year, we hope to include a more generalized treatment of optical and magnetic beam line elements such as apertures, lens, monochromators, etc., and an $R$-matrix treatment of magnetic element transport.

Finally, the present format of GINGER output for use by the post-processor is getting rather long in the tooth. If and when time permits, we hope to evolve this to a more flexible and more efficient format which is "self-describing" (but not in the "classic" SDDS format) and hopefully far reduced in file size. In particular this is needed for temporally "lengthy" beam pulses which are hundreds of cooperation lengths long or greater and for which spectral resolution is needed.

\subsection{Measuring the FEL gain with Trajectory Distortion}

The FEL gain curve can be measured with a trajectory distortion method that stops the FEL interaction near points of these distortions. The method has been demonstrated to be effective in the TTF1 FEL saturation study, and it can be very convenient for the LCLS with a single $\mathrm{x}$-ray diagnostic station installed at the end of the undulator beam line. The FEL fundamental mode at 1.5 $\mathrm{A}$ has an rms divergent angle less of than 1 micro-rad in the exponential gain regime. Both GENESIS 1.3 simulation and theoretical analysis show that a 6 micro-rad angular kick is sufficient to stop further growth of the FEL fundamental mode. This angular kick can be generated, for example, by offsetting a quadrupole center by 60 microns, with the maximum betatron oscillation amplitude of about 120 microns. One potential problem with this method is the increased 
background seen by the end diagnostic station, which includes the spontaneous undulator radiation emitted over the entire undulator length as well as some residual FEL interactions with higher transverse modes. A better signal-to-noise ratio can be gained with angular collimation and far-field diagnostics.

\subsection{A Quick Note on the Possible Usefulness of Coherent Microbunching as a Diagnostic in the Early Stages of the LCLS Undulator}

William M. Fawley, LBNL

Following the discussion during the workshop on the difficulty of picking up the coherent (FEL) signal out of the spontaneous emission, I checked one of my previously done GINGER LCLS SASE runs which illustrated my claim that the coherent bunching in a narrow bandwidth quickly comes out of the general noise background. I think my assertion is well-founded.

The example a run was done a few years ago for the then standard LCLS with a driftfree FODO lattice, $1.2 \mathrm{~mm}$-mrad normalized emittance. The FEL power saturates in about $85 \mathrm{~m}$ at a level of $20 \mathrm{GW}$ and has a gain length of $\sim 4.4 \mathrm{~m}$. The peak of the timeaveraged microbunching is about 0.37 .

A couple years ago I implemented a diagnostic within GINGER which would calculate the spectrum of the instantaneous microbunching $b(\omega)$ at a given z. This is possible because GINGER diagnoses both the amplitude and phase of the instantaneous bunching $b(t)$. At visible wavelengths, the emitted intensity of coherent optical transition radiation will be proportional to $b^{2}(\omega)$. I have average the spectra over four bins (which is still much smaller than the RMS bandwidth. By the $9.5 \mathrm{~m}$ position the bunching in a $1 \%$ bandwidth is nearly double the incoherent bunching and by $15.7 \mathrm{~m}$ the coherent signal to noise ratio in a $0.6 \%$ bandwidth is better than $10: 1$. So, all the collective we need to do is figure out a robust (and inexpensive way) to measure the bunching in a narrow bandwidth at $\mathrm{x}$-ray wavelengths without significant problems arising from the incoherent spontaneous radiation.

Analytically, one can predict the relative growth of the microbunching by employing the dispersion relation for the various modes which predicts a growing mode, a decaying mode, and a purely oscillatory mode. At one gain length in the power, the enhancement in COTR is $(2 \cosh (0.5)+1)^{2} / 9=1.18$. At two gain lengths, the enhancement is 1.85 . At three and four gain lengths it is 3.6 and 8.1, respectively. So presuming we can pick an appropriate bandpass and do some shot-to-shot averaging, in "theory" one should pick up the enhanced COTR by two gain lengths in power from the beginning of the undulator.

\subsection{A Concept For z-Dependent CXTR Microbunching Diagnostics for the LCLS}

Alex Lumpkin, ANL

During the LCLS Diagnostics and Commissioning Workshop held at UCLA on January 19-20, 2004, the group discussed a series of issues with the conflicting constraints of the intra-undulator x-ray diagnostics for the high power mode of the SASE FEL. In particular: 
1) the high absorption of $x$-ray power for the full operating conditions was projected to melt any material at $15 \AA$ and all above carbon at $1.5 \AA$;

2) the beam energy jitter of $0.1 \%$, or $0.2 \%$ in wavelength, compared to the bandwidth of $10^{-5}$ for a diamond crystal monochromator proposed for the SASE diagnostics seemed like a mismatch; and

3) the high level of the spontaneous emission radiation (SER) for a 3\% bandwidth (BW) was projected to mask the SASE radiation in the first $40 \mathrm{~m}$ of the undulator string. Of course in a $0.3 \%$ BW the SASE would be apparent sooner in $\mathrm{z}$.

During the workshop, Bill Fawley mentioned the 37\% microbunching fraction calculated by GINGER at saturation and followed with a short note on 1/21/04 (see section 4.8) on the narrowing spectral bandwidth of microbunching by the $15.8-\mathrm{m}$ point in the undulators.

He left it as an "exercise to the reader" to come up with a way to measure the bunching in a narrow BW at x-ray wavelengths in the presence of SER and SASE. Several of us had discussed at the workshop the need for spectral, spatial, angular, or attenuation filters for sorting the sources to detect the z-dependent FEL performance. I have come up with a combination of concepts that should provide a look at the microbunching evolution. A start-to-end simulation including the diagnostic (S2ED) would be very helpful.

We propose adapting our coherent optical transition radiation (COTR) techniques tested in the VUV-, UV-, and visible regimes at APS [1,2] to the coherent $\mathrm{x}$-ray transition radiation (CXTR) regime. However, our simple use of a thin foil to block completely the much stronger SASE and SER signals compared to UV-Visible COTR is not practical for the $\mathrm{x}$-ray beams. We instead will use the sorting of spectral, angular distribution, and possibly polarization effects to separate the CXTR from the SASE and SER signals. We use the fact that CXTR will be off-axis in an annulus around the SASE, but not redshifted like SASE or SER at that angle. In addition, we may be able to boost the XTR by a decade-old technique. The features of the diagnostic technique are summarized below. We assume that low $-\mathrm{z}$ foils can survive in the 1.5- $\AA$ regime as reported at the workshop by Yang, or a lower-power commissioning beam might be used.

1. Conversion mechanism: The transition radiation generated at the boundary of a material and the vacuum as the e-beam transits the interface is the basis of the technique. Although the yield is lower in the x-ray regime than the visible regime there are still photons, and for a single foil we have two interfaces producing $\mathrm{x}$ rays in the forward direction. The signal goes as the square of the number of interfaces if the thickness is an integral phase step so we gain a factor of 4 . The foil would be inserted at the $0-\mathrm{m}, 10-\mathrm{m}, 20-\mathrm{m}$ station, etc. to sample the beam. One would expect about $10^{4}$ photons in a $1 \% \mathrm{BW}$ at $\sim 8 \mathrm{keV}$ from a carbon foil and $1 \mathrm{nC}$ beam (D.Rule). In this case, we assume the foil-induced scattering or energy straggling will not drastically reduce the microbunching fraction. This should be checked.

2. Resonant X-ray Transition Radiation (RXTR): To boost the XTR signal, we propose evaluating the RXTR technique developed by a number of laboratories a decade or more ago $[3,4]$ to make an $\mathrm{x}$-ray source based on relativistic e-beams transiting a foil 
stack with $\mathrm{M}$ foils. They demonstrated that by choosing the thickness and the separations in integral phase steps, they could enhance the radiation angular density by $\mathrm{M}^{2}$. We need to evaluate if one can scale the beam energies by 50 and still make a realistic (compact) radiator at $8 \mathrm{keV}$. Typical radiators used a few-micron thick foils with separations of 4-48 microns to enhance the 2- to 6-keV x-rays. The sharpness of the annulus of RXTR depends partly on $M$, and the angle of course on 1/gamma and the material plasma frequencies.

3. Annular crystal: At a downstream position ( $+20 \mathrm{~m}$ from the foil for $14.3 \mathrm{GeV}$ ), we would have an annular crystal to interact with the off-axis XTR concentrated in a ring at a radius of $\sim 600 \mu \mathrm{m}$. This crystal would Bragg-select the 1.5-Angstrom x-rays to be directed with $99 \%$ efficiency in its BW $\left(\sim 2 \times 10^{-4}\right.$ for Ge or $\left.\mathrm{Si}\right)$ to the $\mathrm{x}$-ray detector. The on-axis SASE, SER, and e-beam would go through the on-axis hole in the crystal. The off-axis SASE or SER would be redshifted $\sim 15 \%$ by the $\gamma^{2} \theta^{2}$ term (at $30 \mu \mathrm{rad}$ ) of the FEL resonance condition and not satisfy Bragg's condition. A very significant enhancement of the signal to background ratio should result. The off-axis SASE intensity would be down four orders of magnitude at a position $4 \sigma$ away, and the Bragg condition should be selective against the red shifted wavelengths by that amount as well. To address the $0.1 \%$ BW regime, we would need to investigate the use of mosaic crystals.

4. CXTR: In addition, the microbunching of the e-beam in the SASE process would enhance the CXTR signal at $1.5 \AA$ by several orders of magnitude by the $100-\mathrm{m}$ point. The z-dependence of the growth of the CXTR should be measurable in principle, if the sorting aspects work. Details of the coherence length and the effective number of electrons radiating coherently need to be addressed.

5. X-ray detection: The detection of the $\mathrm{x}$ rays could be with an area detector or perhaps a YAG:Ce crystal with an imaging system. Some candidates are in the CDR. We estimate generation of $10^{4}$ to $10^{5}$ or so x-ray photons per 1-nC micropulse without microbunching.

6. Other issues: Foil survivability, mosaic or bent crystals for larger BW, commissioning beam charge and rep rate, another wavelength, beam effects on annulus symmetry and shape, debunching, divergence and pointing, polarization, parametric X-rays, etc. are topics to consider. It would be very instructive to use SPPS for tests of foils, radiators, annular crystal, and detector to establish the XTR source strength near $8 \mathrm{keV}$.

7. Feasibility: I would like to proceed with a full feasibility study to develop the concepts in more detail and to design and perform the prototype experiments as warranted.

\section{References}

1. A.H. Lumpkin et al., Phys. Rev. Lett. Vol. 86, No. 1, 79, January 1, 2001 
2. A.H. Lumpkin et al., Phys. Rev. Lett. Vol. 88, No. 23, 234801-4, June10, 2002

3. M.L. Ter-Mikaelian, High Energy Electromagnetic Processes in Condensed Media, (Wiley-Interscience, New York, 1972)

4. M.A. Piestrup et al., Phys. Rev. A, vol. 45, no.2, 1183, 15 January 1992. (as one example)

\section{Workshop Recommendations}

The workshop was presented with a proposal for commissioning diagnostics and with procedures to carry out FEL commissioning towards the end of the LCLS construction phase. The workshop accepted the general plan. The charge to the workshop was summarized in the question: Will the undulator diagnostics serve commissioning and operations needs for the LCLS? There were concerns and recommendations for a number of items that are described in the following subsections

\subsection{No Intra-Undulator-Segment X-Ray Diagnostics in Baseline Design}

During the presentations it became apparent that there is no solution for $\mathrm{x}$-ray diagnostics to go into the long break sections between undulator segments. The problem is the high $\mathrm{x}$-ray absorption rate at lower photon energies. These diagnostics components carry a high risk. The workshop recommends seeking alternate means of providing the functionality that the inter-undulator diagnostics was supposed to provide. The Trajectory Distortion Method combined with x-ray diagnostics down-stream of the undulator appears to be the most promising.

\subsection{X-Ray Diagnostics Downstream of the Undulator}

There are ideas for workable solutions for x-ray diagnostics at the end of the undulator, where protection from high radiation densities can be provided through attenuators or by positioning the diagnostics components at large distances, i.e. towards the Far Experimental Hall. The main diagnostics components to be used for FEL commissioning downstream of the undulator are a CCD camera (9 microns pixel resolution, $1024 \times 1024$ pixels area) and a spectrometer. The use of these diagnostics components in conjunction with the Trajectory Distortion Method addressed in the next section appears to be sufficient for characterizing the FEL radiation along the undulator.

\subsection{Trajectory Distortion Method to Characterize FEL Radiation vs. z}

In order to be able to measure FEL radiation characteristics, such as power and spectrum as a function of position along the undulator, without having $\mathrm{x}$-ray diagnostics available along the undulator, a method of orbit distortion could be used. This method uses the fact that FEL gain is turned off if the electron beam is forced onto a largeamplitude betatron trajectory. Initial simulations have been carried out and show that with a transverse displacement of one of the quadrupoles by a distance well within the range of quadrupole motion, FEL gain can be stopped and the FEL intensity at the end of 
the undulator is not much different from that at the position of the displaced quadrupole. The x-ray diagnostics downstream of the undulator, (see above) can then be used to characterize the radiation. This method appears feasible. The sensitivity to FEL radiation due to the increased level of spontaneous radiation at the end of the undulator will only be slightly reduced.

\subsection{Roll-Away Undulators}

The option of (remotely or locally) removing each undulator independently transversely from the beam path would allow the characterization of FEL Radiation vs. $\mathrm{z}$ without the interference of extra spontaneous radiation after position $\mathrm{z}$ by removing every undulator down stream of position $z$. This option also offers the potential of characterizing individual undulator segments or pairs of undulator segments through the analysis of the spontaneous radiation that they produce. Z. Huang assessed the possibility of using two consecutive undulator segments to measure their relative phasing and found that that method might work. The use of radiation from a single undulator to assess the quality of the undulator is not promising because of the large radiation bandwidth from a single undulator ( $\mathrm{N}$ 112). The engineering effort for this option is considerable while the potential payoff is small. This option is not recommended by the workshop.

\subsection{Variable Gap Undulators}

A conventional variable gap undulator design constitutes yet another option that would allow us to turn radiation off after any undulator segment in the line by opening the gap of all consecutive undulators. A variable gap undulator could also be used for insitu K correction and for tapering of the undulator line. However, an LCLS prototype undulator has already been successfully developed based on a fixed gap design. Assuming the K set-ability problem can be solved for the fixed gap undulator (and this seems to be the case), the additional cost and time required to develop a variable gap undulator with very close gap tolerances does not seem necessary at this time. This option is not recommended by the workshop.

\subsection{Spontaneous Radiation as Diagnostics Tools}

The workshop strongly recommended exploring the analysis of spontaneous radiation to characterize and improve the undulator line. If none of the methods of turning off radiation from a given $\mathrm{z}$ location on, such as remotely-controlled roll-away undulators or variable gap undulators, can be used the options of characterizing spontaneous radiation are limited. Still it is necessary to perform the necessary computer calculation to generate detailed maps of the near and far field distributions of the spontaneous radiation as seen at an upstream detector.

\subsection{Commissioning in Stages}

The idea of installing the undulator line in two or more parts and commissioning each part with beam before continuing with the installation of the next part has been discussed at the workshop put because of scheduling concerns is not considered at present. 


\subsection{Software Commissioning Support}

It is important to develop software that can predict and analyze the field distribution expected from the LCLS undulator line. Start-to-end codes are already capable of predicting the distribution of the FEL radiation. The existing codes are presently not capable of predicting the full spectrum of spontaneous radiation. The workshop recommended focusing on producing data exchange interfaces between the start-to-end codes and additional radiation analysis codes that might be developed by the x-ray optics groups either from scratch or based on existing $\mathrm{x}$-ray diagnostics codes.

\subsection{Areas for Follow-Up R\&D}

Some areas for follow up R\&D that were identified at the workshop are listed in this section:

- Development of an understanding of the spontaneous radiation pattern

o For the ideal case

o In the presence of undulator errors and misalignments

- Diagnostics Prototyping

o Cavity BPMs

Produce at least three Cavity BPM prototypes and install them in the SPPS beamline in a row with no magnetic components in between.

o Wire Monitor System

Setup a WPS test configuration.

o Gas Attenuator

o Imaging Detector

o Spectrometer

o Solid Attenuator

- Microbunching Measurement 


\section{List of Participants}

John Arthur, Stanford Linear Accelerator Center

Richard Bionta, Lawrence Livermore National Laboratory

Eric Lind Bong, Stanford Linear Accelerator Center

Paul Emma, Stanford Linear Accelerator Center

William M. Fawley, Lawrence Berkeley National Laboratory

John Galayda, Stanford Linear Accelerator Center

Zhirong Huang, Stanford Linear Accelerator Center

Patrick Krejcik, Stanford Linear Accelerator Center

Alex Lumpkin, Argonne National Laboratory

Steven Milton, Argonne National Laboratory

Alex Murokh, University of California at Los Angeles

Pietro Musumeci, University of California at Los Angeles

Heinz-Dieter Nuhn, Stanford Linear Accelerator Center

Claudio Pellegrini, University of California at Los Angeles

Mark Reichanadter, Stanford Linear Accelerator Center

Sven Reiche, University of California at Los Angeles

Robert Ruland, Stanford Linear Accelerator Center

S. Joshua Stein, Argonne National Laboratory

Gil Travish, University of California at Los Angeles

Dean Walters, Argonne National Laboratory

James J. Welch, Stanford Linear Accelerator Center

Bingxin Yang, Argonne National Laboratory 


\begin{tabular}{lll}
$\begin{array}{ll}\text { Workshop } \\
\text { Monday, January 19, 2004 (Knudsen }\end{array}$ & \\
\multicolumn{2}{l}{ Mall) } \\
09:00-09:40 & John Galayda, SLAC & Welcome and Charge \\
09:40-09:45 & Claudio Pellegrini, UCLA & Welcome \\
09:45-09:15 & Heinz-Dieter Nuhn, SLAC & Workshop Issues \\
10:15-10:30 & James Welch, SLAC & Decisions to Make, Questions to Answer \\
10:30-11:45 & Patrick Krejcik, SLAC & Status of Electron Beam Diagnostics \\
11:45-11:55 & & Coffee \\
11:55-13:05 & Steve Milton, ANL & Status of X-Ray Beam Diag. along Und. \\
& Bingxin Yang, ANL & \\
13:05-13:55 & & Lunch Break \\
13:55-14:55 & Richard Bionta, LLNL & X-Ray Diagnostics after the Undulator \\
14:55-15:05 & & Discussion \\
15:05-16:00 & Paul Emma, SLAC & Electron Beam Control and Alignment \\
16:00-16:10 & & Discussion \\
16:10-16:25 & Alex Lumpkin, ANL & X-ray Shutter Concept and Off-axis \\
& & Harmonic Signal Concept \\
16:25-16:55 & & Discussion \\
16:55-17:25 & John Galayda, SLAC & Closing Statement \\
17:25 & & Adjourn
\end{tabular}

\section{Tuesday, January 20, 2004 (Faculty Center)}

$\begin{array}{lll}\text { 09:00-10:30 } & \text { Sven Reiche, UCLA } & \text { Comm. Simulations using GENESIS } 1.3 \\ \text { 10:30-10:40 } & & \text { Discussion } \\ \text { 10:40-11:00 } & & \text { Coffee } \\ \text { 11:00-11:30 } & \text { William Fawley, LBNL } & \text { Comm. Simulations using GINGER } \\ \text { 11:30-11:40 } & & \text { Discussion } \\ \text { 11:40-11:55 } & \text { Zhirong Huang, SLAC } & \text { Measuring the FEL gain w. Trajectory Dist. } \\ \text { 11:55-12:00 } & & \text { General Discussion } \\ \text { 12:00-13:15 } & & \text { Lunch Break } \\ \text { 13:15-14:00 } & \text { John Galayda, SLAC } & \text { Summary and Final Discussion } \\ \text { 14:00 } & & \text { Adjourn }\end{array}$

